\title{
The New Mathematical Model of Motion Compensation for Stepped-Frequency Radar Signal
}

\author{
Yun Lin, ${ }^{1}$ Xiaochun Xu, ${ }^{1}$ Jinfeng Pang, ${ }^{1}$ Bin Li, ${ }^{1}$ and Ruolin Zhou $^{2}$ \\ ${ }^{1}$ Harbin Engineering University, Room 148, Building 21, NO. 145 Natong Street, Nangang District, Harbin, Heilongjiang 150001, China \\ ${ }^{2}$ Department of Electrical and Computer Engineering, Western New England University, Springfield, MA, USA \\ Correspondence should be addressed to Yun Lin; linyun@hrbeu.edu.cn
}

Received 24 August 2013; Revised 28 November 2013; Accepted 9 December 2013; Published 8 January 2014

Academic Editor: Su-Qun Cao

Copyright (c) 2014 Yun Lin et al. This is an open access article distributed under the Creative Commons Attribution License, which permits unrestricted use, distribution, and reproduction in any medium, provided the original work is properly cited.

\begin{abstract}
When a stepped-frequency radar is used to obtain the high-resolution range profile (HRRP) of high-speed target, accurate speed estimation and motion compensation must be considered. Therefore, in this paper, a novel mathematical method is presented for estimating the target speed. Firstly, the pulse Doppler method is used to calculate the initial estimation value. Secondly, based on the initial estimation value, the minimum entropy method is used to calculate the coarse estimation value. Finally, based on the coarse estimation value, the minimum $l_{1}$-Norms method is used to calculate the accurate estimation value. The numeric simulation results confirm that this new method is effective and predominant, which has a much higher estimation accuracy in a low SNR and a much larger estimation range of target speed. The final estimation value can be used to well compensate for the influence of target speed on HRRP.
\end{abstract}

\section{Introduction}

Stepped-frequency (SF) signal is widely used in the highresolution radar systems [1-3], because it can be used to get the high-resolution range profile (HRRP) of target. However, SF signal is highly sensitive to targets motion. The radial speed between target and radar contributes influence on phase term, which will lead to severe range-speed coupling $[4,5]$. As it is well known, the impact of range-speed coupling is well mitigated through motion compensation. Thus, for noncooperative targets, speed estimation method is very important and necessary. In the literature [6,7], based on time domain and waveform entropy, two speed estimation methods are presented. However, they have low estimation accuracy and are only effective in a high signal-to-noise ratio (SNR) and low-speed moving targets. In the literature [8], based on SF and pulse Doppler radar signal, an effective algorithm of speed estimation is presented. Simulation results show that this method has a higher estimation accuracy and better antinoise performance. However, it will increase the complexity of radar system. So far, besides these methods mentioned above, many other effective speed estimation methods have been presented, such as in the literature [9-11]. These methods have gotten much better estimation accuracy. Nevertheless, in common, they have much higher computation complexity and need larger calculation burden; therefore, they are not applicable to use in engineering application. Furthermore, the speed estimation of superspeed moving targets in extremely low SNR is still a key problem for SF signal. And much easier implementation in engineering application also should be taken into account. According to the discussion above, in this paper, a new speed estimation method is proposed. It is a joint speed estimation algorithm, which has a combination of initial, coarse, and accurate estimation process. Simulation experiments have proved that this new method has much higher estimation accuracy and lower calculation burden. Meantime, the effect of HRRP is much better than that used to be for superspeed moving targets in extremely low SNR. Therefore, it can be applied in engineering application.

This paper is organized as follows. Section 1 is the introduction. In Section 2, the mathematic model of SF signal is introduced. In Section 3, the speed estimation algorithm of SF signal is illustrated, and then several comparison experiments 
between this new algorithm and traditional algorithm are designed. In Section 4, the conclusions and future work are described in detail.

\section{The Mathematical Model of SF Signal}

2.1. The Mathematical Model of SF Signal. The SF radar system transmits a series of frames of pulses. Each frame contains a sequence of $N$ pulses with carrier frequencies increasing from pulse to pulse. The carrier frequencies are indicated by $f_{n}=f_{0}+n \Delta f$, where $n=0,1, \ldots, N-1, f_{0}$ is the fundamental carrier frequency, and $\Delta f$ is the frequency step. Thus, the total bandwidth is $B=N \Delta f$. The transmitted SF pulse train during the integration time is described as follows:

$$
S_{T}(t)=\sum_{m=0}^{M-1} \sum_{n=0}^{N-1} \operatorname{rect}\left(\frac{t-t_{m, n}}{T_{p}}\right) \exp \left[-j 2 \pi\left(f_{0}+n \Delta f\right) t\right]
$$

where

$$
\operatorname{rect}\left(\frac{t-t_{m, n}}{T_{p}}\right)= \begin{cases}1, & 0 \leq \frac{t-t_{m, n}}{T_{p}} \leq 1 \\ 0, & \text { otherwise }\end{cases}
$$

with $t_{m, n}=m T_{f}+n T_{r}, m=0,1, \ldots, M-1 ; n=0,1, \ldots, N-1$. $M$ is the frame number and $N$ is the pulse number in each frame. $\operatorname{rect}(t)$ is the envelope of the transmitted pulse and is assumed to be normalized to unity during the pulse duration $T_{p} . T_{r}$ is the pulse repetition interval and $T_{f}$ is the frame period. $T_{N}=N T_{r}$ is the duration time of SF pulse train, which can be used to represent the processing time required to form the HRRP. The illustration of SF signal is shown in Figure 1.

Suppose that the moving target is composed of a set of $P$ points scatters with backscattering coefficients $A(p)$, with $p=1,2, \ldots, P$. Therefore, the received signal from a moving target can be expressed in (3) as follows:

$$
\begin{aligned}
S_{R}(t)= & \sum_{p=1}^{P} A(p) S_{T}\left[t-\tau_{p}(t)\right] \\
= & \sum_{m=0}^{M-1} \sum_{p=1}^{P} \sum_{n=0}^{N-1} A(p) \operatorname{rect}\left[\frac{t-t_{m, n}-\tau_{p}(t)}{T_{1}}\right] \\
& \times \exp \left[-j 2 \pi\left(f_{0}+n \Delta f\right)\left(t-\tau_{p}(t)\right)\right],
\end{aligned}
$$

where $\tau_{p}(t)$ is the echo delay of the pth scattering point. In this paper, in order to simplify the analytic process, the acceleration and rotation of target are ignored. Therefore, the $\tau_{p}(t)$ is shown in

$$
\tau_{p}(t)=\frac{2 R_{p}(t)}{c}=\frac{2\left[R_{p}(0)-v t\right]}{c},
$$

where $R_{p}(0)$ is the radial distance of $p$ th scatter point to radar at the initial time, $v$ is the radial speed of moving target, and $c$ is the speed of light.
2.2. The Mathematic Analysis of SF Signal in Moving Environment. In general, during each frame period, the change of envelope with the target motion can be neglected. Therefore, after the down conversion and sampling of the SF receivedsignal, the baseband signal is shown in

$$
\begin{aligned}
& S_{D}(m, n) \\
& =\sum_{p=1}^{P} A(p) \exp \left\{j 2 \pi\left(f_{0}+n \Delta f\right)\right. \\
& \left.\times \frac{2\left[R_{p}(0)-\left(m T_{f}+n T_{r}\right) v\right]}{c}\right\} \\
& =\sum_{p=1}^{P} A(p) \exp \{j 2 \pi \phi(m, n)\} .
\end{aligned}
$$

In this paper, the number of points for inverse discrete Fourier transform (IDFT) is $N$, the HRRP of target can be calculated by (6) with the $m$ th frame SF received signal:

$$
\begin{aligned}
g(m, i) & =\sum_{n=1}^{N} S_{D}(m, n) \exp \left\{j 2 \pi \frac{i n}{N}\right\} \\
& =\sum_{n=1}^{N} \sum_{p=1}^{P} A(p) \exp \{j 2 \pi \phi(m, n)\} \exp \left\{j 2 \pi \frac{i n}{N}\right\} .
\end{aligned}
$$

Note that the HRRP of target is calculated only by every single frame; therefore, the influence of target motion on HRRP is only embodied in every single frame. In order to simplify the analytic process, suppose $m=0$. According to (5) and (6), the influence of target motion on HRRP is embodied in the phase $\phi(0, n)$, which is shown in

$$
\begin{aligned}
\phi(0, n) & =\left(f_{0}+n \Delta f\right) \frac{2\left[R_{p}(0)-v n T_{r}\right]}{c} \\
& =\underbrace{\frac{2 f_{0} R_{p}(0)}{c}}_{\text {const }}+\underbrace{\frac{2 n R_{p}(0) \Delta f}{c}}_{f_{R}}-\underbrace{\frac{2 f_{0} v T_{r} n}{c}}_{f_{d}}-\underbrace{\frac{2 v \Delta f T_{r} n^{2}}{c}}_{\text {Spread }} .
\end{aligned}
$$

Equation (7) reveals the special properties of SF signal. For stationary targets, only const and $f_{R}$ are applicable. The first term represents a constant phase migration, which has no practical influence on HRRP. The second term is produced by the product of frequency. Therefore, the distance between target and radar is converted into the frequency migration, which can be used to calculate the distance between target and radar. In general, the frequency migration can be calculated by IDFT. Furthermore, the range resolution $\Delta r$ and unambiguous range $R_{u}$ are, respectively, dependent on frequency resolution and maximum unambiguous frequency of IDFT. The expression for these quantities is described in

$$
\Delta r=\frac{c}{2 B}=\frac{c}{2 N \Delta f}, \quad R_{u}=\frac{c}{2 \Delta f} .
$$




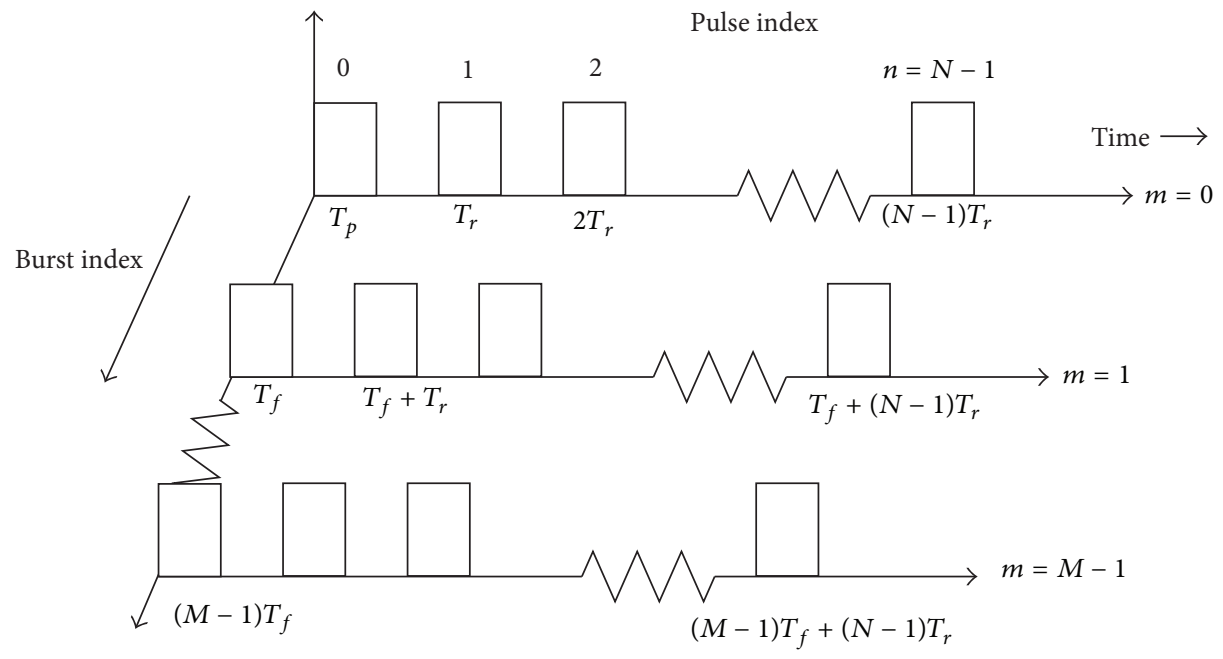

FIgURE 1: Illustration of $M$ frames of SF Signal.

The third term $f_{d}$ in (7) is a linear phase term (LPT), which represents the Doppler frequency migration due to target motion. The processing of IDFT mistakes the Doppler frequency as a frequency migration due to range and thus results in the shifting of target range from its true range. According to the literature [5] and (8), suppose, in order to perfectly compensate for LPT, that the sustainable error of distance measurement is half of range resolution of SF signal, which is shown as follows:

$$
\Delta R=\frac{\Delta r}{2}=\frac{c}{4 N \Delta f} .
$$

Then, the estimation accuracy of target speed must satisfy

$$
|\Delta v|_{\mathrm{LPT}} \leq \frac{c}{2}\left(\frac{\Delta f}{f_{0}}\right)\left(\frac{1}{T_{r}}\right)\left(\frac{1}{N \Delta f}\right)=\frac{c}{4 f_{0} N T_{r}} .
$$

The fourth term "Spread" in (7) is a quadratic phase term (QPT), which causes the frequency spread. This phase will disperse target energy into several range bins and lead to several negative effects including the loss of range resolution, range accuracy, and signal-to-noise radio. According to the literature [5], suppose, in order to perfectly compensate for QPT, the sustainable change of QPT satisfies the following requirement:

$$
2 \pi \frac{2 N \Delta f|\Delta v|_{\mathrm{QPT}}}{c} N T_{r} \leq \frac{\pi}{2} .
$$

Then, the estimation accuracy of target speed must satisfy

$$
|\Delta v|_{\mathrm{QPT}} \leq \frac{c}{8 N^{2} \Delta f T_{r}} .
$$

According to the discussion above, the compensation accuracy of LPT is much higher than that of QPT. Therefore, the LPT needs an accurate speed estimation and the QPT only needs a coarse speed estimation. When the target speed is estimated, according to (5) and (7), the LPT and QPT can be eliminated.
TABLE 1: The main parameters of SF radar signal.

\begin{tabular}{lccccc}
\hline$f_{0}$ & $N$ & $\Delta f$ & $T_{1}$ & $T_{r}$ & $M$ \\
\hline $94 \mathrm{GHz}$ & 128 & $4 \mathrm{MHz}$ & $100 \mathrm{~ns}$ & 20 us & 96 \\
\hline
\end{tabular}

TABLE 2: The main performance of SF radar signal.

\begin{tabular}{lccc}
\hline$\Delta r$ & $R_{u}$ & $|\Delta v|_{\mathrm{LPT}}$ & $|\Delta v|_{\mathrm{QPT}}$ \\
\hline $0.3 \mathrm{~m}$ & $37.5 \mathrm{~m}$ & $0.308 \mathrm{~m} / \mathrm{s}$ & $28.610 \mathrm{~m} / \mathrm{s}$ \\
\hline
\end{tabular}

In this paper, the main parameters of SF signal are shown in Table 1, and the main performance parameters of SF signal are shown in Table 2.

In this paper, let us suppose that the target speed is constant and takes no account of its own rotation. Meanwhile, the maximum radial length of the target is less than $R_{u}$. There are four strong radial scatters which are shown in Figure 2 $(R=2 \mathrm{~km})$, and the radio of normalized scattering intensity (NSI) is $1: 10: 6: 8$.

\section{The Speed Estimation Algorithm of SF Signal}

According to the discussion above, the influence of target motion on HRRP is embodied in the additional LPT and QPT. In order to get better HRRP, firstly, the target speed must be estimated, and then the additional phase terms need to be eliminated. In this paper, based on some traditional mathematic model, a new accurate and fast speed estimation algorithm is proposed.

3.1. The Pulse Doppler Algorithm (PDA). This method is based on pulse Doppler effect; firstly, through IDFT with SF received signal, the Doppler frequency can be calculated, and then the target speed can be calculated by

$$
\widehat{v}_{\mathrm{IE}}=\frac{2 f_{0} f_{d}}{c} .
$$




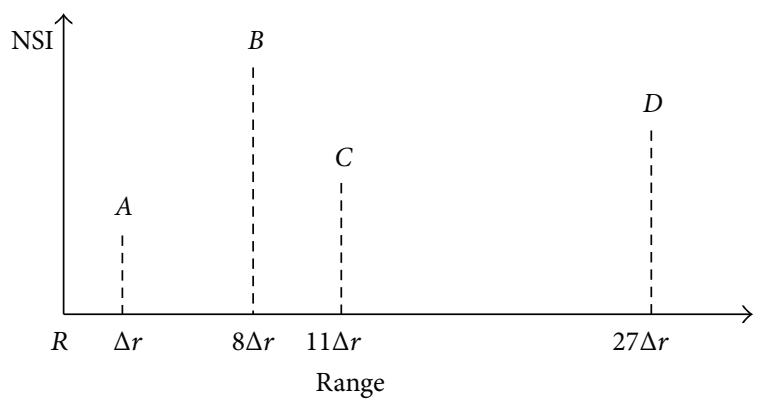

FIGURE 2: The model of target.

According to the IDFT theory, the speed estimation accuracy of PDA is shown in

$$
\Delta v_{\mathrm{IE}}=\frac{\Delta f_{d} c}{2 f_{0}}=\frac{c}{2 M f_{0} T_{r}} .
$$

In this paper, the accuracy of speed estimation $\Delta \widehat{v}_{\mathrm{IE}}$ is less than $0.831 \mathrm{~m} / \mathrm{s}$, which is only enough to compensate for the QPT. In addition, because of the maximum unambiguous frequency caused by the IDFT, PDA has a great estimation limitation to the interval of target speed, which is shown in (15)

$$
v_{\max }=\frac{c}{2 f_{0} T_{r}} .
$$

Here, $v_{\max }=79.787 \mathrm{~m} / \mathrm{s}$; therefore, if the target speed goes over $v_{\max }$, the estimation result will satisfy (16) and cause much greater error:

$$
v_{\mathrm{PDA}}=\widehat{v}_{\mathrm{IE}}+k v_{\max } \quad k=1,2, \ldots
$$

Figure 3 indicates the estimation error of PDA in different speed (within an unambiguous rang of speed estimation) and SNR by Monte Carlo experiments; the experiments time is 1000. This simulation experiment indicates that PDA is effective to compensate for QPT in low SNR, but it has a great limitation to $v_{\max }$.

3.2. The Minimum Entropy Algorithm (MEA). It is well known that the overall target response in the HRRP is changing with the target motion, such as the reduction of peak response, the divergence of scatters, and the blurring effects. According to the theory of image processing, the entropy is a measure of the randomness of an image. The focused images will have a low entropy values. Thus, the mathematical model of entropy can be used as a quality indicator for HRRP. It is defined by

$$
H_{m}=-\sum_{i=0}^{N-1} \widehat{g}_{m}(i) \operatorname{In} \widehat{g}_{m}(i) \quad \widehat{g}_{m}(i)=\frac{|g(m, i)|}{\sum_{i=0}^{N-1}|g(m, i)|} .
$$

According to Formula $(6), g(m, i)$ can be obtained by the IDFT. If the target motion is well compensated, the entropy of HRRP will be minimized. In summary, this method uses the entropy of HRRP as evaluation function to find target speed, because the estimation value will minimize the entropy of HRRP. Supposing that the speed of target is $V=2000 \mathrm{~m} / \mathrm{s}$, Figure 4 shows the entropy feature of HRRP in different speed, which proves the effectiveness of MEA. In addition, the MEA is a search process, which needs an initial speed and an effective search algorithm (such as simulated annealing, evolutionary, and genetic algorithm).

Figure 5 indicates the estimation error of MEA with different speed and SNR by Monte Carlo experiments, and the experiments time is also 1000. This simulation experiment proves that, with the SNR decreasing, the estimation accuracy of EMA will rapidly decline, and it can only be used to compensate for QPT in low SNR. However, the EMA has a much bigger interval of speed estimation than that of PDA.

3.3. The Minimum $l_{1}$-Norms Algorithm (MNA). This algorithm needs to transmit two different frames and each frame contains $N$ pulses, which is shown in Figure 6 . The first frame is the up frame, the carrier frequency at each pulse increases from $f_{0}$ to $f_{0}+(N-1) \Delta f$. The second frame is the down frame, the carrier frequency at each pulse descends from $f_{0}+(N-1) \Delta f$ to $f_{0}$. Therefore, the frequency of $n$th pulse in up-frame can be calculated by $f_{n}^{1}=f_{0}+n \Delta f$ and in downframe can be calculated by $f_{n}^{2}=f_{0}+(N-n-1) \Delta f$.

According to (5), the $n$th pulse of received signal can be described as

$$
\begin{gathered}
S_{D}^{1}(n)=\sum_{p=1}^{P} A(p) \exp \left\{j 2 \pi f_{n}^{1} \frac{2\left[R_{p}(0)-v n T_{r}\right]}{c}\right\}, \\
S_{D}^{2}(n)=\sum_{p=1}^{P} A(p) \exp \left\{j 2 \pi f_{n}^{2} \frac{2\left[R_{p}(0)-v(N+n) T_{r}\right]}{c}\right\} .
\end{gathered}
$$

Compensating with a initial speed $\widehat{v}_{\mathrm{IE}},(18)$ is converted into the following form:

$$
\begin{gathered}
S_{\mathrm{DC}}^{1}(n)=\sum_{p=1}^{P} A(p) \exp \left\{j 2 \pi f_{n}^{1} \frac{2\left[R_{p}(0)-\Delta v n T_{r}\right]}{c}\right\}, \\
S_{\mathrm{DC}}^{2}(n)=\sum_{p=1}^{P} A(p) \exp \left\{j 2 \pi f_{n}^{2} \frac{2\left[R_{p}(0)-\Delta v(N+n) T_{r}\right]}{c}\right\},
\end{gathered}
$$

where $\Delta v=v-\widehat{v}, S_{\mathrm{DC}}^{1}(n)$ and $S_{\mathrm{DC}}^{2}(n)$ represent a coarse compensation with the initial target speed. Then, the function of frame error can be defined as $l_{1}$-Norms in

$$
R_{\varphi}(\Delta v)=\frac{1}{N} \sum_{n=0}^{N-1}\left|S_{\mathrm{DC}}^{1}(n)-S_{\mathrm{DC}}^{2}(n)\right|,
$$

and then it can be further simplified as

$$
R_{\varphi}(\Delta v)=\frac{1}{N} \sum_{n=0}^{N-1}\left|\sin \left[2 \pi T_{r}\left(f_{0}+n \Delta f\right)(2 N-1-2 n) \frac{\Delta v}{c}\right]\right| .
$$




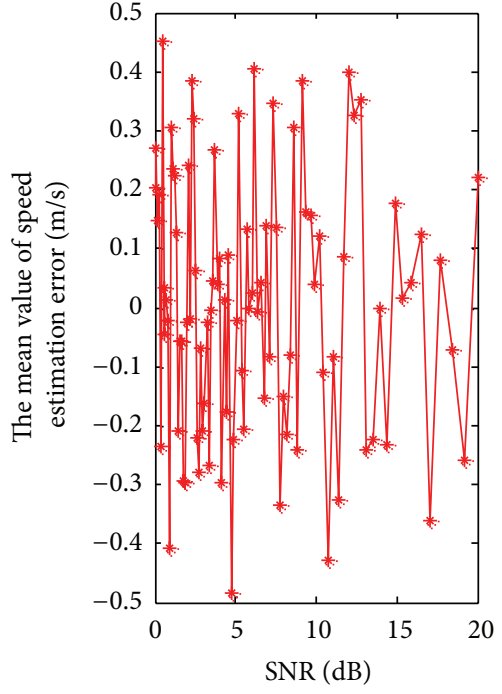

(a)

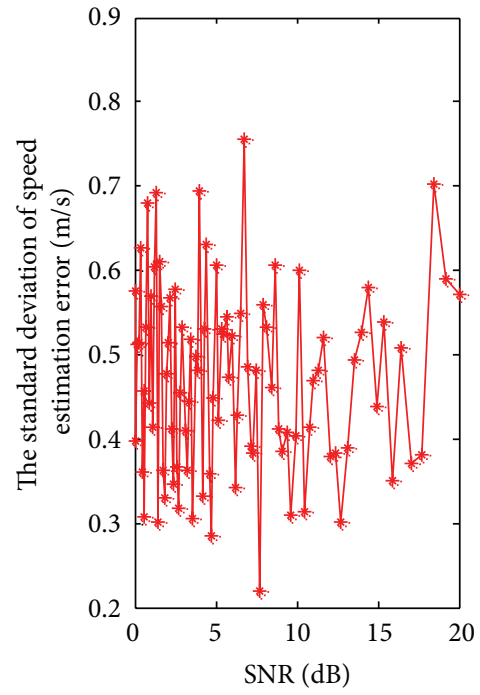

(b)

FIgURE 3: The estimation error of PDA.

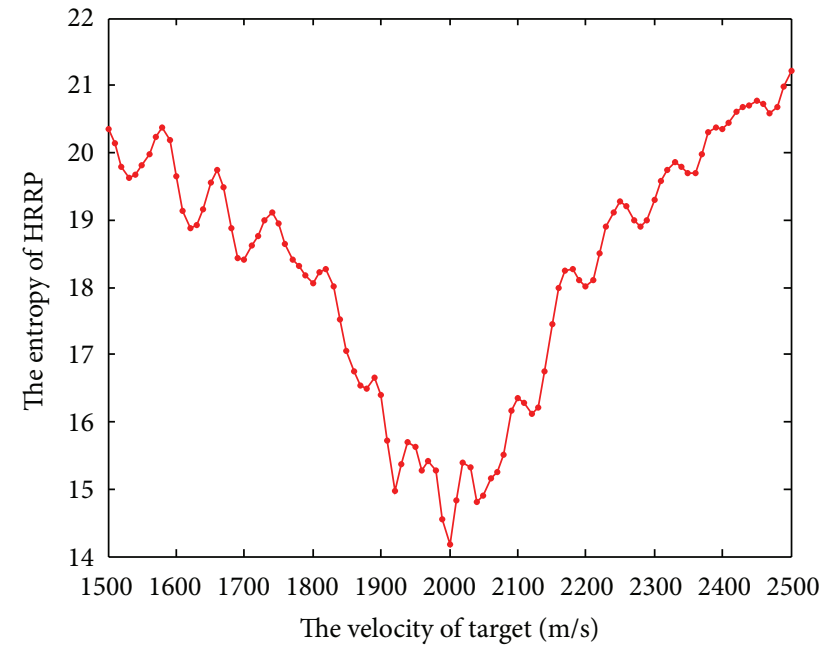

Figure 4: The entropy feature of MEA.

According to (21), $\Delta v$ makes a difference between $S_{\mathrm{DC}}^{1}(n)$ and $S_{\mathrm{DC}}^{2}(n)$ which can be used to estimate target speed. If the target motion is well compensated, then it satisfies $R_{\varphi}(\Delta v)=0$. Considering the complexity of target motion and signal environment, the evaluation function of global optimal estimation can be defined as

$$
R_{\varphi}(\widehat{v})=\min \left\{R_{\varphi}(\Delta v)\right\} .
$$

Supposing that the speed of target is $V=2000 \mathrm{~m} / \mathrm{s}$, Figure 7 shows the value of frame error in different speed, which proves the effectiveness of MNA. Therefore, target speed estimation can be performed by searching the minimum peak in Figure 7. Within the searching procedure, the following problems should be considered.
(1) When the initial estimation value is chosen, wrong searching direction will prolong searching time and increase computation amount.

(2) In order to get global minimum value as accurate as possible, search step size should be less than the width of the peak. Therefore, the computation amount may be significantly increased.

(3) Even if the SNR of received signal is high, the curve of evaluation function may also have some local fluctuation nearby the true value. Therefore, if the search algorithm is not very effective, the evaluation function may converge to the local minimum which will cause much higher estimation error.

Figure 8 indicates the estimation error of MNA in different speed and SNR by Monte Carlo experiments; the experiments time is also 1000, and the search step of MNA is $1 \mathrm{~m} / \mathrm{s}$. This simulation experiment proves that the estimation accuracy of MNA is very high than that of PDA and MEA, and it can be used to compensate results in LPT in very low SNR.

3.4. The Compound-Estimation Algorithm (CEA). According to the discussion above, in order to overcome the defects of PDA, MEA, and MNA, an improved speed estimation algorithm is presented. It contains three steps including initial estimation, coarse estimation and accurate estimation.

The SF radar system transmits alternately the up frame and the down frame and the received signals are shown in

$$
\mathbf{S}_{D}=\left[\begin{array}{cccc}
S_{D}(1,1) & S_{D}(1,2) & \cdots & S_{D}(1, N) \\
S_{D}(2,1) & S_{D}(2,2) & \cdots & S_{D}(2, N) \\
\vdots & \vdots & \vdots & \vdots \\
S_{D}(M, 1) & S_{D}(M, 2) & \cdots & S_{D}(M, N)
\end{array}\right] .
$$




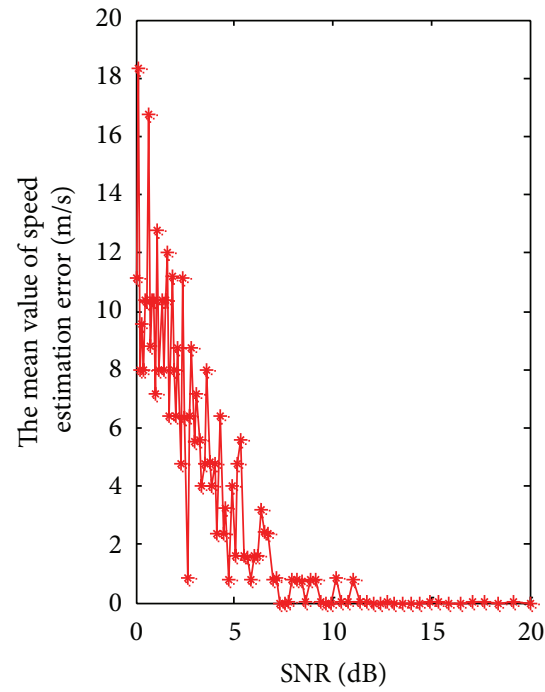

(a)

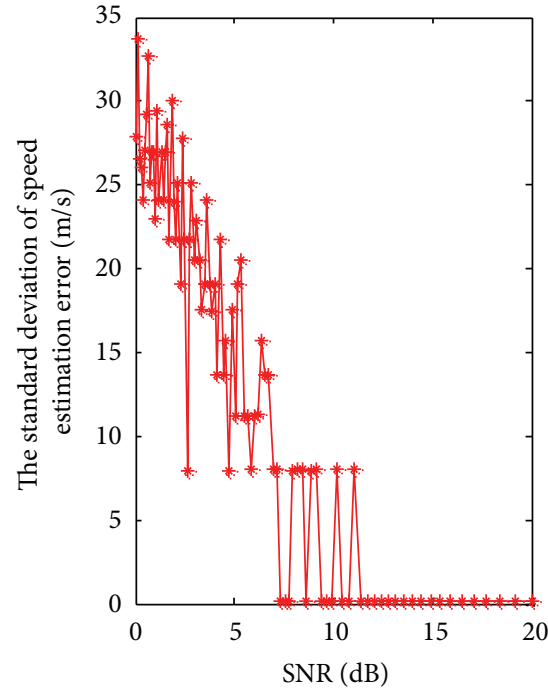

(b)

FIGURE 5: The estimation error of MEA.

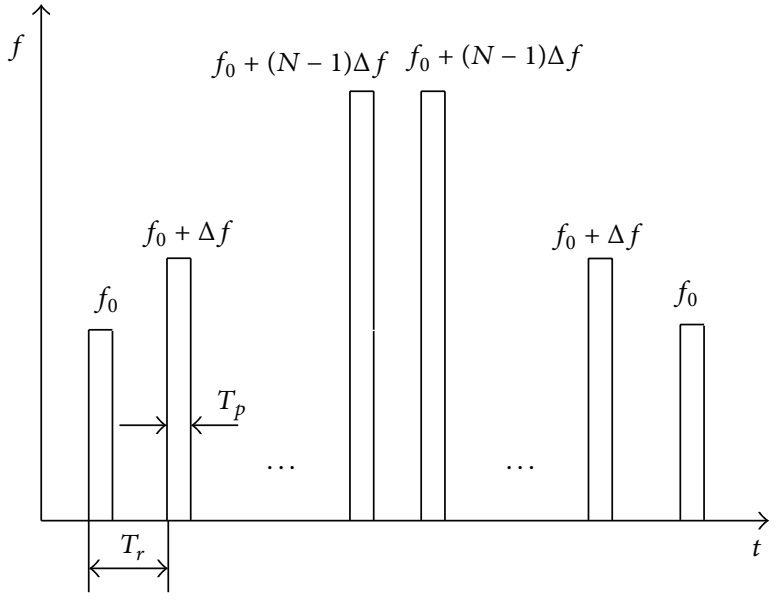

Figure 6: The model of the up frame and results in frame.

The odd row is the up-frame and the even row is the downframe. The value of $M=2 L$ is even.

3.4.1. The First Step: Initial Estimation (IE) Based on PDA. PDA is used for initial estimation with the first column in (23). The new signal sequence for estimation is shown in

$$
\begin{gathered}
\mathbf{S}_{D}^{\text {odd }}=\left[\begin{array}{llll}
S_{D}(1,1) & S_{D}(3,1) & \cdots & S_{D}(2 L-1,1)
\end{array}\right], \\
\mathbf{S}_{D}^{\text {even }}=\left[\begin{array}{lllll}
S_{D}(2,1) & S_{D}(4,1) & \cdots & S_{D}(2 L, 1)
\end{array}\right] .
\end{gathered}
$$

According to the discussion in Section 3.1, the estimation value can be calculated by (13) and (24) with IDFT, and they are defined as $\widehat{v}_{\text {IE }}^{\text {odd }}$ and $\widehat{v}_{\mathrm{IE}}^{\text {even }}$. Then, the initial estimation value can be calculated by

$$
\widehat{v}_{\mathrm{IE}}=\frac{\widehat{v}_{\mathrm{IE}}^{\mathrm{odd}}+\widehat{v}_{\mathrm{IE}}^{\mathrm{even}}}{2} .
$$

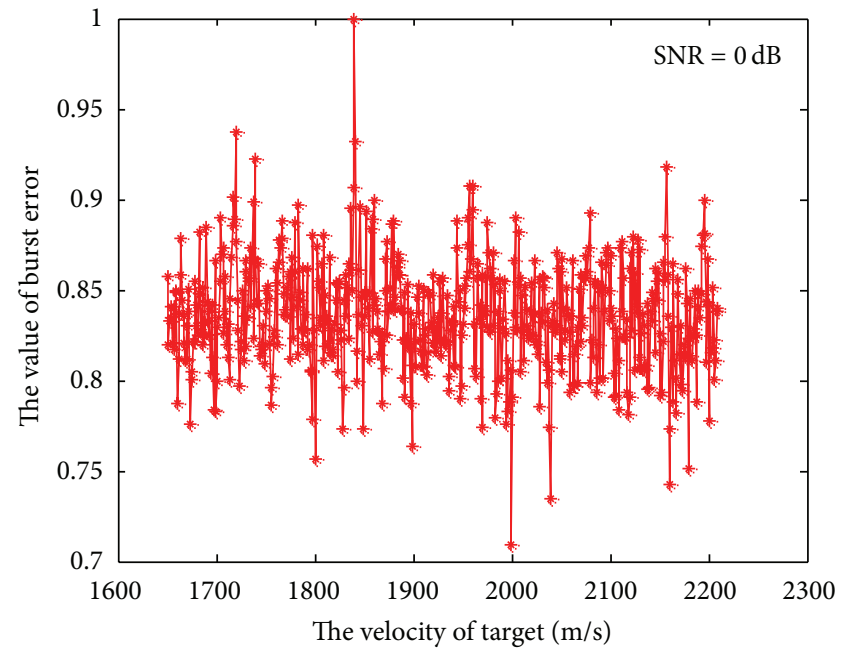

Figure 7: The frame error feature of MNA.

In this paper, according to the main parameters of SF signal in Table 1, the initial estimation error is $\left|\Delta \widehat{v}_{\mathrm{IE}}\right| \leq$ $1.662 \mathrm{~m} / \mathrm{s}$, which is only enough to compensate for the QPT. The maximum unambiguous estimation interval of target speed is $\left|\widehat{v}_{\text {IE }}\right| \leq v_{\max }=79.787 \mathrm{~m} / \mathrm{s}$. In this paper, suppose that the estimation interval of target speed is $V \in[0,6000] \mathrm{m} / \mathrm{s}$; therefore, according to (16), there are a total of 77 possible estimation values, which are $k=0,1, \ldots, 76$. Therefore, the MEA is used for expanding the estimation interval of target speed.

3.4.2. The Second Step: Coarse Estimation (CE) Based on MEA. After compensating with initial estimation value, MEA is used for coarse estimation with the first and second row 


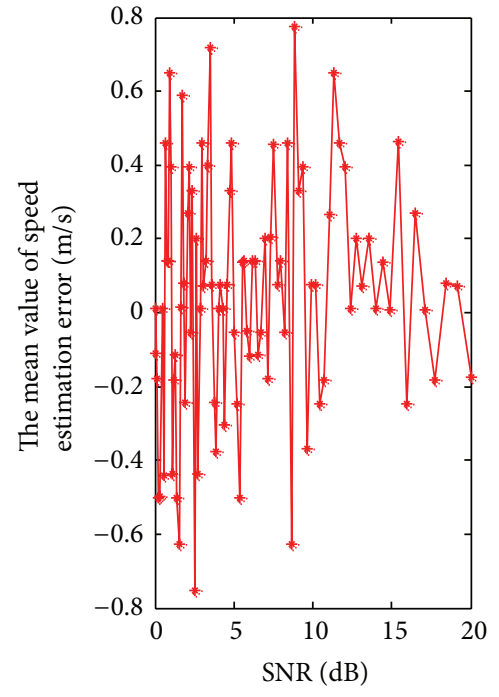

(a)

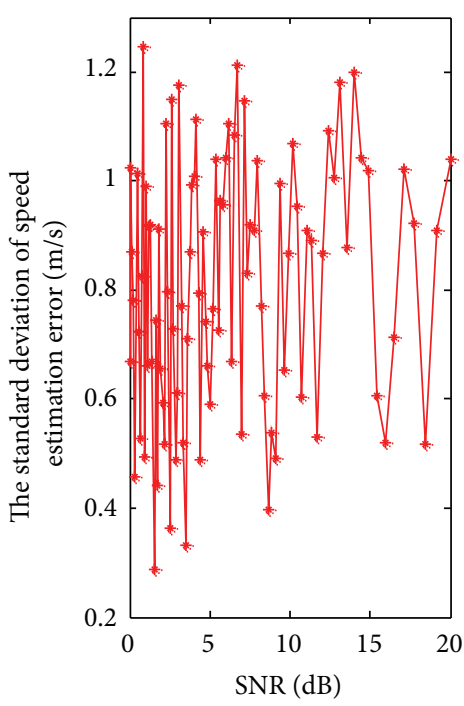

(b)

Figure 8: The estimation error of MNA.

in (23). The new SF signal sequence for estimation is shown in

$$
\begin{aligned}
& \mathbf{S}_{D}^{1}=\left[\begin{array}{llll}
S(1,1) & S(1,2) & \cdots & S(1, N-1)
\end{array}\right] \\
& \mathbf{S}_{D}^{2}=\left[\begin{array}{llll}
S(2,1) & S(2,2) & \cdots & S(2, N-1)
\end{array}\right] .
\end{aligned}
$$

According to the discussion in Sections 3.1 and 3.2, the coarse estimation values can be calculated by (17) and (26) with these initial estimation values, which are shown as follows:

$$
v_{\mathrm{CE}}^{i}=\widehat{v}_{\mathrm{IE}}+k v_{\max } \quad k=0,1, \ldots, 76, i=1,2 .
$$

According to these 77 possible values, two coarse estimation values which are defined as $\widehat{v}_{\mathrm{CE}}^{i}$ can be calculated, and then the final value of coarse estimation can be calculated by

$$
\widehat{v}_{\mathrm{CE}}=\frac{\widehat{v}_{\mathrm{CE}}^{1}+\widehat{v}_{\mathrm{CE}}^{2}}{2}
$$

According to the discussion of MEA method, the possible value of target speed can be determined. However, this coarse estimation value is only enough to compensate for QPT. Therefore, finally, the MNA is used to increase the estimation accuracy of target speed.

3.4.3. The Third Step: Accurate Estimation (AE) Based on $M N A$. When the coarse estimation value is obtained, the true value of target speed will surely consist in $\left[\widehat{v}_{\mathrm{CE}}-\widehat{v}_{\mathrm{IE}} / 2, \widehat{v}_{\mathrm{CE}}+\right.$ $\left.\widehat{v}_{\mathrm{IE}} / 2\right]$. Therefore, the final estimation value can be defined as $\widehat{v}_{\mathrm{AE}}=\widehat{v}_{\mathrm{CE}}+k \Delta v$, which statisfies $\widehat{v}_{\mathrm{AE}} \in\left[\widehat{v}_{\mathrm{CE}}-\widehat{v}_{\mathrm{IE}} / 2, \widehat{v}_{\mathrm{CE}}+\right.$ $\widehat{v}_{\text {IE }} / 2$ ]. According to the discussion in Table 2 and Section 3.3, in order to compensate for the LPT, choosing the search step as $\Delta v=0.2 \mathrm{~m} / \mathrm{s}$, the MNA is used to calculate the accurate estimation value with (19), (20), and (26), which can be used to compensate for LPT.
Figure 9 indicates the estimation error of CEA in $V \in$ $[0,6000] \mathrm{m} / \mathrm{s}$ and $\mathrm{SNR} \in[0,20] \mathrm{dB}$. It proves that the CEA is effective to compensate for LPT in a much larger estimation interval of target speed and extremely low SNR.

Figures 10 and 13 are the HRRP of a moving target with four different compensation methods. HRRP0 is the HRRP of static target. Figure 10 is the HRRP of a moving target based on PDA. Because of the limitation of estimation interval, the HRRP1 is completely a distortion and cannot identify target. Figure 11 is the HRRP of a moving target based on MEA, and the HRRP2 produces a big range migration beyond an unambiguous range window. In addition, it can be used to identify target. However, because of the limitation of estimation accuracy, the HRRP2 cannot be used for distance measurement. Figure 12 is the HRRP of a moving target based on MNA, and the HRRP3 produces a little migration within an unambiguous range window and also can be used to identify target. The accuracy of distance measurement is much more accurate than that of MEA. Figure 13 is the HRRP of a moving target based on CEA, and the HRRP4 produces the smallest migration within an unambiguous range window and has the highest accuracy of distance measurement; the HHRP4 also can be used to identify target. Therefore, the CEA has the best compensation result for identifying target and distance measurement.

\section{Conclusion}

In this paper, three different speed estimation models are analyzed in detail. The PDA has an accurate estimation value in low SNR. However, it has a large limitation to estimation interval of target speed, which will make a great estimation error for a high-speed moving target. The MNA has a coarse estimation value in a much larger speed estimation interval. However, it has a large limitation to SNR, and the estimation accuracy is not enough for well compensating the influence 


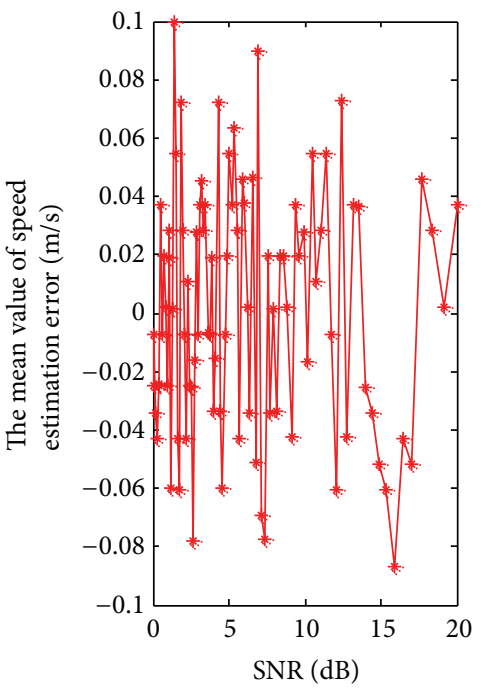

(a)

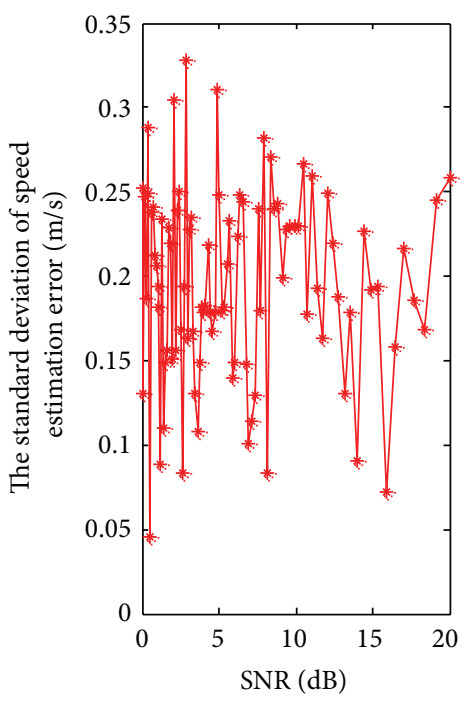

(b)

Figure 9: The estimation error of CEA.

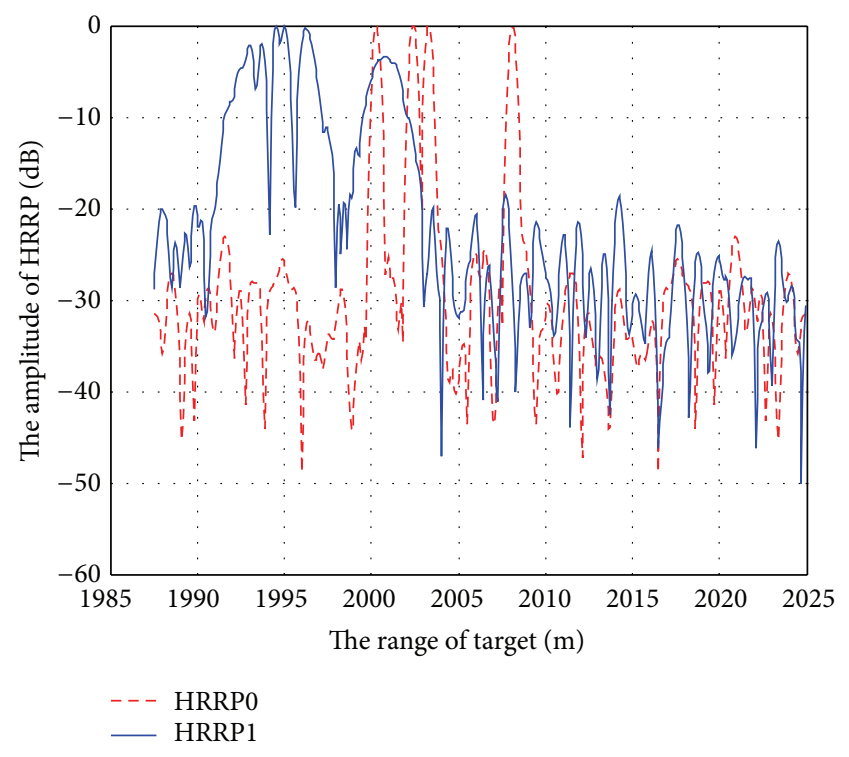

FIgURE 10: The HRRP of SF signal based on PDA.

of target speed on HRRP. The MNA has the best estimation accuracy. However, the local minimum value of frame error function will produce worse estimation accuracy and make a large computation amount.

According to the advantages and defects of PDA, MEA, and MNA, the CEA is presented in this paper. Firstly, according to the main parameters of SF signal, some important performance parameters can be calculated. Secondly, the initial estimation value is obtained by PDA. Thirdly, based on the initial estimation value and the estimation interval of target speed, the MEA is used for the coarse estimation value. Finally, based on the coarse estimation value and the final estimation accuracy, the MNA is used for the accurate

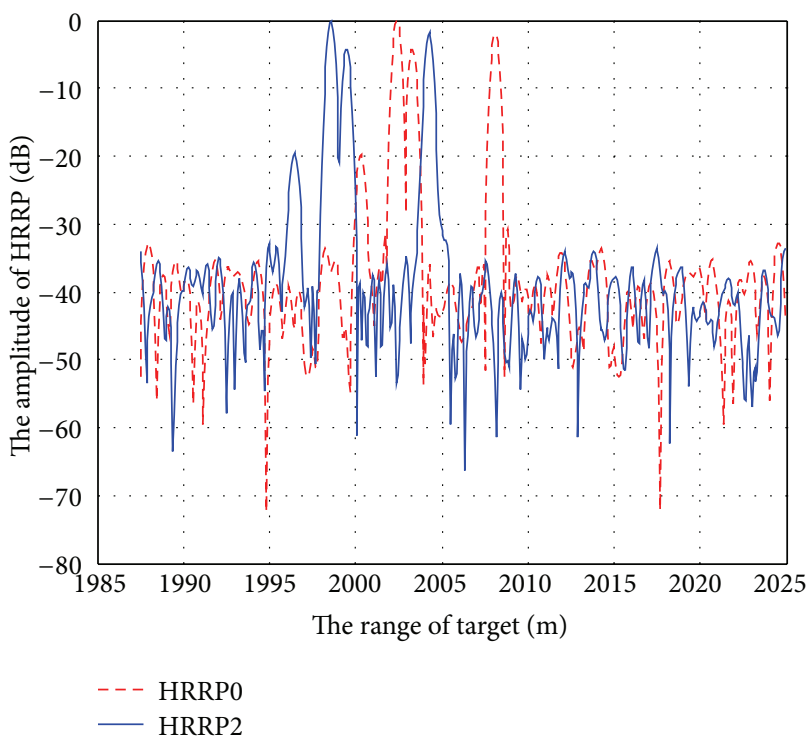

FIGURE 11: The HRRP of SF signal based on MEA.

estimation. The theory analysis and simulation results confirm that this new method is effective and predominant, which has a much higher speed estimation accuracy in realtime and a much larger estimation interval of target speed. Furthermore, the influence of super-high speed motion of target on HRRP can be perfectly compensated, and the HRRP can be used to improve the detection, recognition, and distance measurement performance of moving target.

\section{Conflict of Interests}

The authors declare that there is no conflict of interests regarding the publication of this paper. 


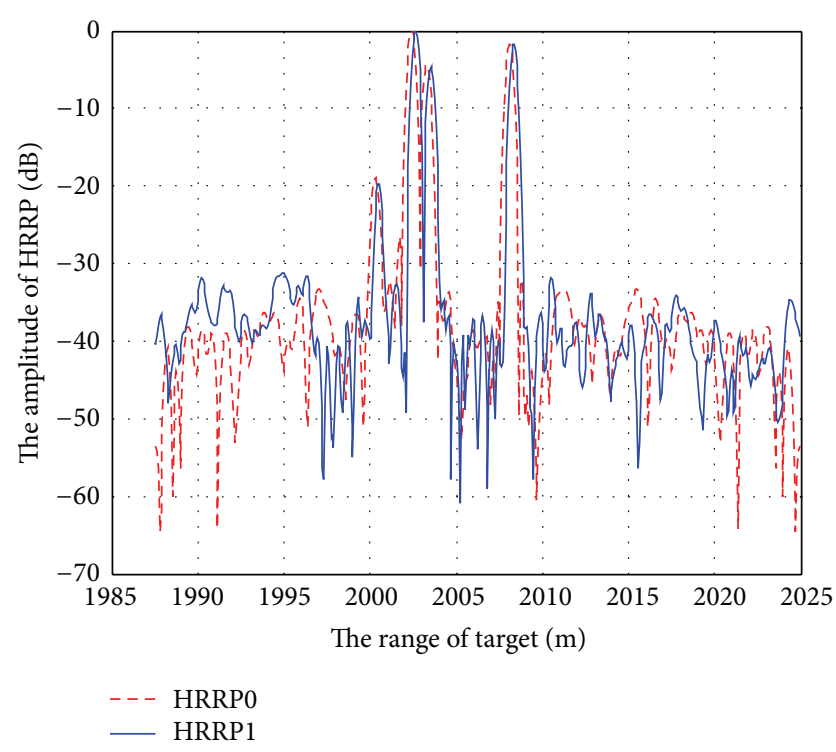

FIGURE 12: The HRRP of SF signal based on MNA.

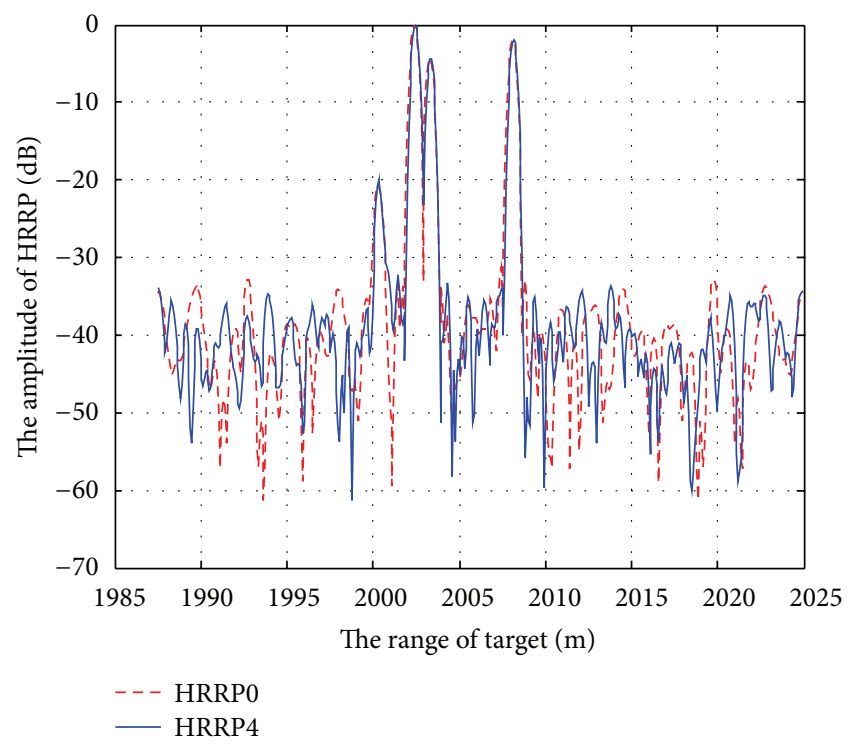

FIgURE 13: The HRRP of SF signal based on CEA.

\section{Acknowledgments}

This work is supported by the Nation Nature Science Foundation of China no. 61301095 and 61201237, Nature Science Foundation of Heilongjiang Province of China no. QC2012C069, and the Fundamental Research Funds for the Central Universities no. HEUCFZ1129, no. HEUCF130810, and no. HEUCF130817.

\section{References}

[1] D. R. Wehner, "Novel range profile synthesis algorithm for linearly stepped-frequency modulated inversed synthetic aperture radar imaging of remote manoeuvring target," Let Radar Sonar and Navigation, vol. 5, no. 4, pp. 496-506, 2011.
[2] T. H. Einstein, "Contrast-optimization-based range-profile autofocus for polarimetric stepped-frequency radar," IEEE Transactions on Geoscience and Remote Sensing, vol. 48, no. 4, pp. 2049-2056, 2010.

[3] B. Pang, D.-H. Dai, S.-Q. Xing et al., "Imaging enhancement of stepped-frequency radar using the sparse reconstruction technique," Progress in Electromagnetics Research-Pier, vol. 140, pp. 63-89, 2013.

[4] S. Chang-gui and L. Xing-guo, "Two-step stretch processing method for MMW high-resolution radar," Journal of Infrared and Millimeter Waves, vol. 22, no. 6, pp. 457-460, 2006.

[5] L. Teng, "Doppler performance analysis of frequency stepped radar signal," Modern Radar, vol. 2, pp. 31-37, 1996.

[6] L. Jing, L. Xing-guo, and W. Wen, "Application of waveform entropy method for motion compensation of MMW Costas frequency hopped radar," Journal of Infrared and Millimeter Waves, vol. 22, no. 4, pp. 303-306, 2003.

[7] J. Liu, X.-G. Li, and Y.-H. Li, "Motion compensation for 1$\mathrm{d}$ range profile of moving target in MMW Costas frequency hopped radar," Journal of Infrared and Millimeter Waves, vol. 24, no. 5, pp. 344-347, 2005.

[8] W. Gui and L. Xing-guo, "Compound approach of measuring speed based on step-frequency and pulse doppler system," Journal of Infrared and Millimeter Waves, vol. 27, no. 3, pp. 191194, 2008.

[9] F. Berizzi, M. Martorella, A. Cacciamano, and A. Capria, "A contrast-based algorithm for synthetic range-profile motion compensation," IEEE Transactions on Geoscience and Remote Sensing, vol. 46, no. 10, pp. 3053-3062, 2008.

[10] G. Xia, H. Su, and P. Huang, "Velocity compensation methods for LPRF modulated frequency stepped-frequency (MFSF) radar," Journal of Systems Engineering and Electronics, vol. 21, no. 5, pp. 746-751, 2010.

[11] L. Hai-bo and L. Jun-dao, "Target motion compensation algorithm based on keystone transform for wideband pulse doppler radar," Transactions of Beijing Institute of Technology, vol. 32, no. 6, pp. 626-631, 2012. 


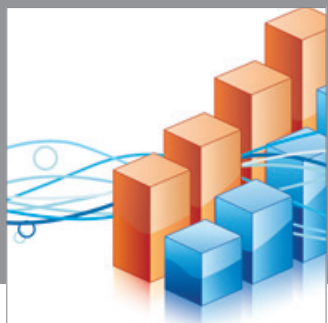

Advances in

Operations Research

mansans

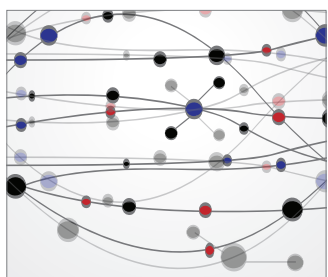

The Scientific World Journal
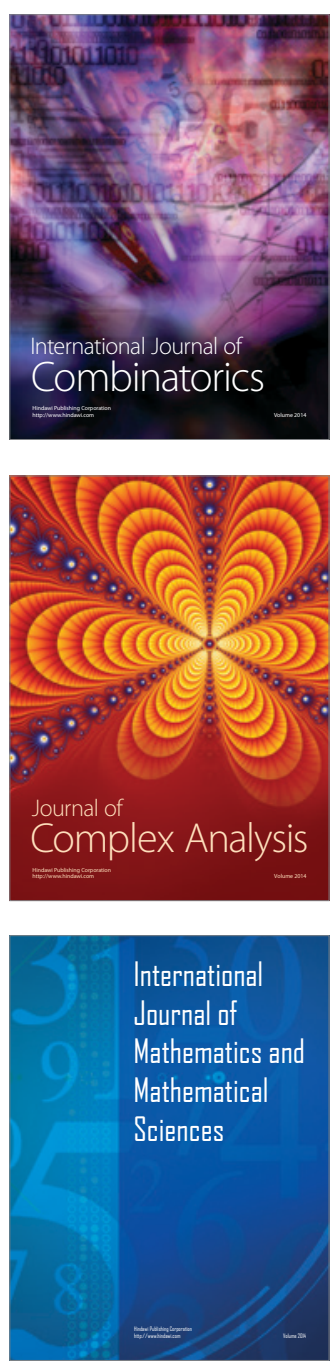
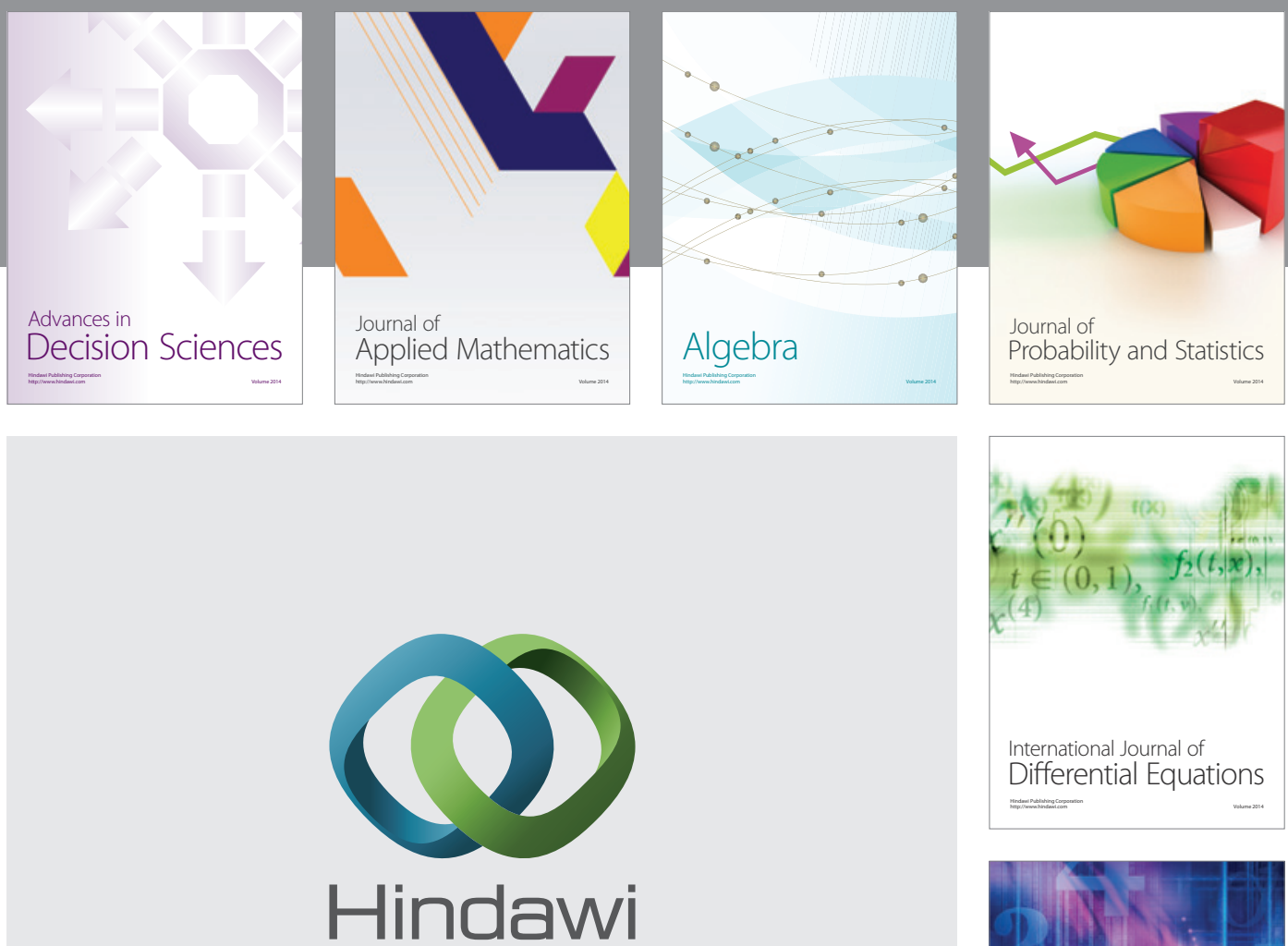

Submit your manuscripts at http://www.hindawi.com
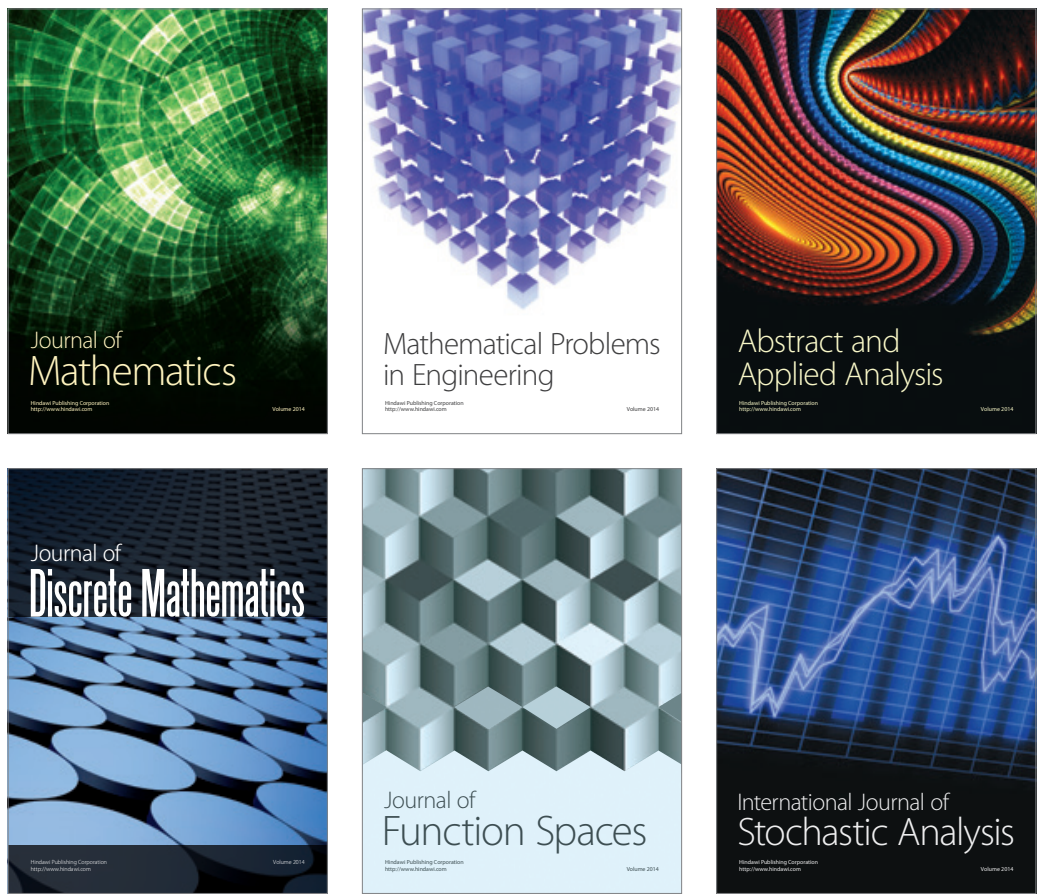

Journal of

Function Spaces



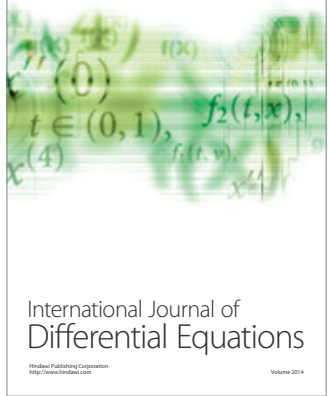
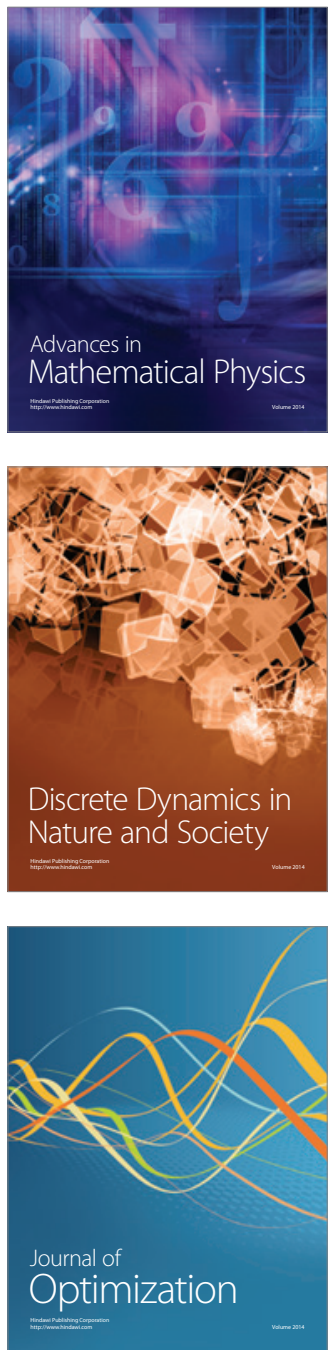